\title{
Testing for a large roundabouts capacity model: experimental comparisons between Italy and Bahrain
}

\author{
A. Pratelli ${ }^{1} \&$ H. M. N. Al-Madani ${ }^{2}$ \\ 'Dipartimento di Ingegneria Civile "Vie e Trasporti", \\ University of Pisa, Italy \\ ${ }^{2}$ Department of Civil Engineering and Architecture, \\ College of Engineering, University of Bahrain, Bahrain
}

\begin{abstract}
It is well-known that the maximum traffic flow that a roundabout entry is able to accommodate depends on the amount of flow circulating in the roundabout carriageway that conflicts with the entry flow exiting from it and strictly related to the geometric characteristics of the roundabout. International guidelines deal with various capacity models requiring different input data and often lead to different results for the same roundabout entry. Recently, an exponential model has been developed for capacity estimates of multi-lane roundabout entries under different conditions of circulating flow and geometrical factors. Such a model was validated by Al-Madani and Saad on data gathered from a sample of existing large roundabouts in Bahrain. Calculations made, applying the developed model, or BAHR model, showed a reasonable fitting to computational data obtained from different international models, as German, French SETRA, American $\mathrm{HCM}$, and so on.

In this paper, we have collected traffic data during peaks or congested periods and measured geometry parameters on twelve large roundabouts located in Tuscany, Italy. The main goal was with a view to testing if the BAHR model could be well suited or not to Italian context. The same previous methodology applied for the BAHR model was followed and found a new exponential entry capacity model, called TUSC. Statistical tests are performed and the paper ends with some comments about the obtained results.
\end{abstract}

Keywords: roundabout capacity, linear regression and exponential entry capacity models, statistical significance. 


\section{Introduction}

During the Sixties, soon after the United Kingdom and following many other Commonwealth countries, all the traffic circles in Bahrain were converted into modern roundabouts adopting the priority-to-the-circle rule. Since about thirty years after the modern roundabouts began to appear also in Italy.

Nowadays, modern roundabouts are widely spreading all over the world because of their advantages over other types of intersection control. The main reasons are linked to increasing of safety over other cross roads [2] due to a reduction in severity of crashes, and deep improvement of performance, as observed in Europe and the USA after adopting a roundabout in replacement to any other existing type of intersection [3].

At the same time, many countries have developed design guidelines referring to specific models for the capacity evaluation of modern roundabouts. All the capacity models can be classified into two groups. The first one is related to theoretical models based on the gap acceptance theory, with no actual observations. The second group collects empirical regression models, derived on the basis of large samples both of actual observed geometric characteristics and surveyed data of traffic flow parameters. This way, the designer often might fall into a dilemma. On one side there are the theoretical models supporters, as Fisk [4], asserting that regression models are not suited for frequent application due to their large number of data requirements. On the opposite side it is said, as Kimber [5] does, that capacity estimates based on gap acceptance models are not suitable for application in original nationwide contexts due to the amount of factors related to local driver' behaviour and habits.

Two years ago Al-Madani and Saad [1] developed an exponential model for capacity estimate of roundabouts with triple circulating lanes. The model was derived in respect to the entry capacity values obtained from a selected set of international models applied to actual data observed on thirteen large roundabouts in Bahrain.

This paper deals with a first attempt of following the same methodological steps in order to develop an original model on data now gathered from a sample of roundabouts located Tuscany (Italy). Finally, the previous and the present developed models, said BAHR model and TUSC model respectively, were compared through goodness of fit and statistical test procedures in order to state if they can be or not considered the same one.

\section{Capacity models}

This section is referred to six of popular models related to the capacity of roundabouts and widely used from some decades in Western Europe and Australia, and more recently in US also. All they are suitable to predict the entry capacity as many for a single lane entry as for multi-lane entries. The capacity of the entire roundabout is not considered, as it depends on too many terms. The yield line is the relevant point for capacity analysis, and the approach capacity is the capacity provided at the yield line [6]. 
The maximum flow rate that can be accommodated at a roundabout entry depends on three factors: the circulating flow on the circulating roadway that conflicts with the entry flow, the exiting flow on the same leg of the entry, and the geometric dimensions and characteristics of the roundabout. When analyzing roundabout legs with more than one entry lane, the capacity for each lane is different, with the dominant stream having the greater capacity. Doubling the number of entry lanes does not give double the total entry capacity. The performance of multiple lane roundabouts will be improved if the inscribed diameter is increased, and if the average entry lane width is increased [7]. This is because the drivers' gap acceptance parameters are reduced. Therefore, driver behaviour is expected to be different at higher circulating flows where priority sharing occurs. A number of fundamental methods applicable to two-way-stopcontrolled and two-way-yield-controlled intersection capacity analysis serve as a foundation for roundabout operational performance.

Two different methodological approaches have been used to develop such models: gap acceptance theory and empirical regression. In a gap acceptance model, the driver on the minor (entering) stream is required to select an acceptable gap on the major (circulating) stream, to perform the desired manoeuvre. The "gap" is defined as the headway maintained between two consecutive vehicles in the conflicting stream. In order to cope with particular concerns related to gap acceptance in a roundabout environment, Troutbeck and Kako have developed a theory for incorporating a "limited priority" process, in which the major stream vehicle slows down to allow the minor street vehicle to enter the circulating stream [8]. Linear or exponential empirical regression models are based on traffic volumes at one-minute intervals observed during periods of overflow. A well-suited regression equation, usually linear or exponential, is then fitted to the sample data. Variation in the data is often generated by driver behaviour and geometric design. The influence of geometric design is also included into the model using a multivariate regression equation [7]. The international technical literature quotes several both of theoretical and empirical capacity models, often drawn from national guidelines of various countries. Moreover, some models are complicated requiring extensive input data and tedious calculations as Great Britain RODEL, Australians aaSIDRA and NAASRA, others are far more simple and require few input data as German HBS and US Highway Capacity Manual models.

Capacities estimated in respect to the same instance through these models may widely differ from one model to another. The crucial question of how good the capacity estimate of each model is, calls for further investigations, but it is not the goal of this paper.

\subsection{French GIRABASE model}

The model is an exponential regression that takes into account a number of geometric parameters and the influence of exiting flow. It is incorporated into a software implementation known as GIRABASE [9] and its form is as follows:

$$
C_{e}=A \cdot \exp \left(-B \cdot q_{g}\right)
$$


where:

$C_{e}=$ maximum entry flow $(\mathrm{pcu} / \mathrm{h}) ; q_{g}=q_{a} \cdot k_{a} \cdot\left(1-\left(q_{a} /\left(q_{c}+q_{a}\right)\right)\right)+q_{c i} \cdot k_{t, i}+q_{c e} \cdot k_{t, e} ; q_{c i}$ = conflicting flow on inner lane (default $0.4 q_{c}$ ); $q_{c e}=$ conflicting flow on outer lane (default $\left.0.6 q_{c}\right) ; q_{a}=$ exiting flow (pcu/h);A=3600/t $t_{f}\left(L_{e} / 3.5\right)$;

$t_{f}=$ follow-up headway $=2.05 \mathrm{~s} ; B=3.525$ for urban area (3.625 for rural area);

$R=$ radius of the central island $(\mathrm{m}) ; L_{a}=$ circulating roadway width $(\mathrm{m})$;

$L_{e}=$ entry width (m); $L_{i}=$ width of the splitter island (m);

$L_{i, \max }=4.55 \sqrt{R+\left(L_{a} / 2\right)}$; for $L_{i}<L_{i, \max } K_{a}=\left(R /\left(R+L_{a}\right)\right)-\left(L_{i} / L_{i, \max }\right)$; else $K_{a}=0$;

$k_{t, i}=\min \left\{160 /\left(L_{a} \cdot\left(R+L_{a}\right) ; 1\right\} ; k_{t, e}=\min \left\{1-\left(\left(L_{a}-8\right) / L_{a}\right) \cdot\left(R /\left(R+L_{a}\right)^{2}\right)\right) ; 1\right\}$

\subsection{Great Britain model}

The model suggested by Kimber [10] is received into UK guidelines. It is a statistically derived empirical linear formula based on a large number of measurements of capacity at saturated roundabouts.

This method has been incorporated into a software packages widely known as RODEL and ARCADY. The model involves circulating flows and extensive geometric requirements (see also Figure 1), and it has the following form:

$$
C_{e}=F-f_{c} Q_{c}
$$

where:

$F=303 k x ; f_{c}=0.210 k T(1+0.2 x) ; x=v+(e-v) /(1+2 S) ; T=1+0.5 /(1+M)$ $k=1-0.00347(\phi-30)-0.978[(1 / r)-0.05] ; M=\exp [(D-60) / 10] ; S=1.6(e-v) / L$,

The geometric parametrs are: $e=$ entry width $(\mathrm{m}) ; v=$ approach half-width (m); $L^{\prime}=$ effective flare length (m); $r=$ entry radius $(\mathrm{m}) ; S=$ measure of the degree of the flaring; $\phi=$ entry angle $\left(^{\circ}\right) ; D=$ inscribed circle diameter $(\mathrm{m})$. The predictive equation for entry capacity $C_{e}$ is given by equation (3) when the product $f_{c} Q_{c}$ is a value less than or equal to $F$; otherwise $C_{e}=0$.

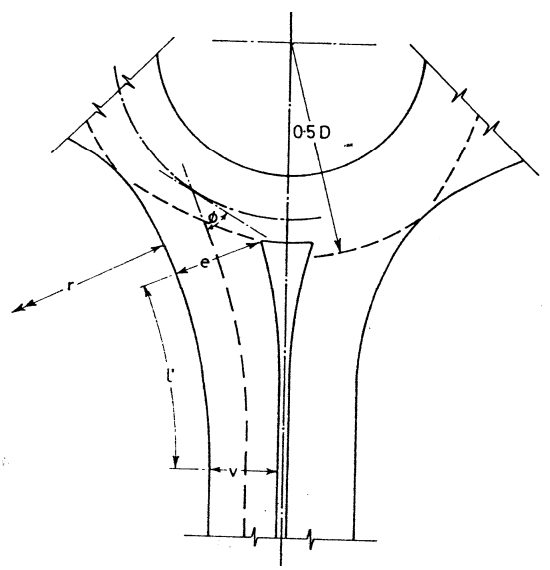

Figure 1: Definition of the geometric requirements in Kimber's equation. 


\subsection{German HBS model}

In 2001, the Brilon-Wu capacity equation has been introduced into the German handbook of highways design and practice, or HBS [11]. This model is an exponential equation relating entry capacity $C_{e}$ to circulating flow $q_{c}$, the number $n_{e}$ of lanes in the entry, the number $n_{c}$ of conflicting lanes ( 1 or 2 with $n_{c} \leq n_{e}$ ) critical gap $t_{c}$ and follow-up headway $t_{f}$ and minimum headway $\Delta$ of circulating stream:

$$
C_{e}=\frac{n_{e}^{n_{F} /\left(n_{F}+1\right)}}{t_{f}} \cdot\left(1-\frac{\Delta \cdot q_{c}}{n_{c}}\right)^{n_{c}} \cdot \exp \left[-q_{c} \cdot\left(t_{c}-\frac{t_{f}}{2}-\Delta\right)\right]
$$

where: $n_{F}=$ short lane length $=1.4$ vehicles; $t_{c}=3.3 \mathrm{~s}, t_{f}=3.1 \mathrm{~s}$ and $\Delta=1.8 \mathrm{~s}$ are the suggested default values.

\subsection{Australian aaSIDRA}

The more recent capacity expressions published in Australia belong to the studies carried out by Akçelik et al. [12] and have been incorporated into the software known as aaSIDRA.

Capacity $C_{e}$ means the maximum entry flow for an entry lane and it is calculated lane-by-lane as follows:

$$
\left\{\begin{array}{l}
C_{e}=\max \left\{f_{o d} q_{g} ; q_{m}\right\} \\
q_{g}=\left[\left(1-\Delta_{c} q_{c}\right) / \beta\right]+0.5 \beta \varphi_{c} q_{c} \exp \left[-\lambda\left(\alpha-\Delta_{c}\right)\right] \\
q_{m}=\min \left\{q_{e} ; n_{m}\right\} \\
f_{o d}=1-f_{q c}\left(P_{q d} P_{c d}\right)
\end{array}\right.
$$

where:

$q_{c}=q_{c r}+q_{c o}=$ inner and outer conflicting flow (veh/s); $q_{e}=$ entry arrival flow $(\mathrm{veh} / \mathrm{s}) ; P_{q d} P_{c d}=0.6 ; n_{m}=$ minimum entry flow $(\mathrm{veh} / \mathrm{s}) ; n_{c}=$ number of lanes in conflicting flow; $\Delta_{c}=$ minimum headway in circulating traffic (s): $\Delta_{c}=2.0$ for $n_{c}=1, \Delta_{c}=1.2$ for $n_{c}=2 ; \lambda=$ arrival headway distribution factor $(\mathrm{veh} / \mathrm{s})$ : for $q_{c} \leq$ $\left(0.98 / \Delta_{c}\right)$ then $\lambda=\left(\varphi_{c} q_{c}\right) /\left(1-\Delta_{c} q_{c}\right)$, else $\lambda=\left(49 \varphi_{c} / \Delta_{c}\right)$; for $n_{c}=1 \varphi_{c}=\exp \left(-5.0 q_{c}\right)$, for $n_{c}=2$ or $3 \varphi_{c}=\exp \left(-3.0 q_{c}\right) ; \beta=$ follow-up headway.

For the dominant entry lane (lane at a multi-lane with the largest entry flow $\left.q_{d}\right): \beta=\beta_{d}=\left(\beta{ }_{o}{ }_{o}-3.94 \cdot 10^{-4} q_{c}\right)$, subject to $1.2 \leq \beta^{\prime}{ }_{d} \leq 4$ $\beta^{\prime}{ }_{o}=3.37-0.0208 D_{i}+0.889 \cdot 10^{-4} D_{i}{ }^{2}-0.395 n_{e}+0.388 n_{c}$, subject to $20 \leq D_{i} \leq 80$ where: $D_{i}=$ inscribed circle diameter $(\mathrm{m}) ; n_{e}=$ number of entry lanes.

For the subdominant entry lane (i.e. lane at a multi-lane with the smallest entry flow $\left.q_{s}\right): \beta=\beta_{s}=2.149+r_{d s}\left(0.5135 \beta_{d}-0.8735\right)$, subject to $\beta_{d} \leq \beta_{s} \leq 4$ where: $r_{d s}=q_{d} / q_{s}=$ ratio of dominant and subdominant flow in the entry; $\alpha=$ critical headway (s): for $q_{c} \leq 1200$ then $\alpha=3.6135-3.137 \cdot 10^{-4} q_{c}-0.339 w-0.2775 n_{c}$, else $\alpha=\beta\left(3.2371-0.339 w-0.2775 n_{c}\right)$, subject to $3.0 \geq \alpha / \beta \geq 1$ and $2.2 \leq \alpha \leq 8.0$; 
$w=$ average entry width $(\mathrm{m})$; if $n_{c}=1$ then: $f_{q c}=0.04+0.00015 q_{c}$ for $q_{c}<600$, $f_{q c}=0.0007 q_{c}-0.29$ for $600 \leq q_{c} \leq 1200, f_{q c}=0.55$ for $q_{c}>1200$; if $n_{c}=2$ then: $f_{q c}=0.04+0.00015 q_{c}$ for $q_{c}<600, f_{q c}=0.0035 q_{c}-0.29$ for $600 \leq q_{c} \leq 1800$, $f_{q c}=0.55$ for $q_{c}>1800$.

\subsection{Australian NAASRA}

The National Association of Australian State Road Authorities, or NAASRA, adopted Tanner's method for delays and capacity of uncontrolled intersections both in the 1979 guidelines to urban intersection design and updated 1982 roundabout guidelines. The model is summarized as follows:

$$
C_{e}=n_{c} q_{c} \frac{\exp \left(-q_{c} t_{c}\right)}{1-\exp \left(-q_{c} t_{f}\right)}
$$

where: $q_{c}=$ circulating flow; $n_{c}=$ number of conflicting lanes; $t_{c}=$ critical interval $=6 \mathrm{~s} ; t_{f}=$ follow-up time $=3 \mathrm{~s}$.

\subsection{United states HCM2000}

After the first introduction in 1997, the 2000 edition of Highway Capacity Manual [13] refers to a method for multi-lane roundabout capacity as described below:

$$
C_{e}=1230 \cdot n_{c} \cdot \exp \left(-0.0009 q_{c}\right)
$$

where, as above: $q_{c}=$ circulating flow; $n_{c}=$ number of conflicting lanes.

\section{Methodology}

Following the methodology suggested by Al-Madani and Saad [1] in their previous work, an original capacity model has been developed on geometric and traffic data observed in twelve large roundabouts located Tuscany (Italy). We named this model TUSC in order of distinguishing from the one developed in Bahrain, and referred as BAHR model in the following. Then, the TUSC model is in its turn an equation linking roundabout entry capacity to the corresponding circulating flow. Sample data were collected during rush hours and under saturation flow conditions. Some different types of models were developed firstly using a least square regression procedure. Secondly, all the resulting linear, logarithmic, polynomial, power and exponential equations were evaluated for best experimental data fitting. Finally, capacities of the twelve Italian roundabouts were calculated using both TUSC model and BAHR one. The two resulting sets were statistically analyzed in order to determinate if they may be considered to have come from the same population, or, in other words, as to whether using TUSC or BAHR model is the same or different in practice. Such hypothesis has been tested by computing of both the differences of means and the homogeneity of variances by the exact F-test. 


\section{Sample data}

Data has been collected on a sample of twelve large roundabout located in the North-Western part of Tuscany, Italy. All over they have the circulating roadway width suited for two lanes operations, while the entries are sometimes with two lanes or one lane only, especially for those sizes falling into the lower range from 40 to 45 metres of inscribed diameter. Traffic flow measurements made during weekday a.m. and p.m. peak hours were related to each single leg. Field traffic data were firstly video recorded by digital camera for about two hours per each selected peak period of interest. Successive analysis of the video recorded images made we able to refer traffic values on the standard 15-minutes basis.

On the other side, geometric parameters were accurately observed recurring to on-field measurements by laser instruments. Few problems of local accessibility were coped with searching for and viewing directly the original building up design layouts from National Highway Agency - ANAS and public road bureaus of local municipalities. Satellite images from Google Earth ${ }^{\mathrm{TM}}$ have also helped to refine and/or improve some of details belonging to the geometric sample set.

Table 1 resumes the main distinctive characteristics of the twelve roundabouts sampled in Tuscany (It).

Table 1: Sample composition of the twelve roundabouts observed in Tuscany.

\begin{tabular}{|c|c|c|c|}
\hline D range $(\mathrm{m})$ & N. of roundabouts & 3 legged & 4 legged \\
\hline $40 \div 45$ & 2 & - & 2 \\
\hline $45 \div 53$ & 3 & - & 3 \\
\hline$>53$ & 7 & 5 & 2 \\
\hline
\end{tabular}

\section{$5 \quad$ TUSC model developing}

The six models quoted above were applied to the sample data. This way a set of capacity values has been generated and processed by a least square method search for the best goodness of data fitting [14].

The performed analysis leads to five different types of models: linear, logarithmic, exponential, quadratic and power regression. Among them, the exponential model shows the best sample data fit when compared with the other models because it holds the highest $R^{2}$ value $\left(R^{2}=0.504\right)$.

Table 2 shows the $R^{2}$ values obtained for each best corresponding model among each one of the five tested forms. It is worthwhile remembering that the model type set up in Bahrain, the said BAHR model, is also exponential as below:

$$
C_{e}=2768.0 \cdot \exp \left(-0.0007 q_{c}\right)
$$

While the selected best fit equation for the TUSC model is:

$$
C_{e}=2272.2 \cdot \exp \left(-0.0009 q_{c}\right)
$$


At the first look, equations (7) and (8) are quite similar. Therefore, it is moved up along this resemblance that the two models were submitted to a test analysis concerning their differences, as referred in the following section.

Table 2: $\quad$ Best fit capacity equations for each one of five model forms obtained in respect to the sample data observed in Tuscany (It.).

\begin{tabular}{|c|c|c|}
\hline Model form & Best fit equation & $R^{2}$ \\
\hline Linear & $C_{e}=2132.6-1.112 q_{c}$ & 0.426 \\
\hline Logarithmic & $C_{e}=3556.9-336.4 \cdot \operatorname{Ln}\left(q_{c}\right)$ & 0.294 \\
\hline Exponential & $C_{e}=2272.2 \cdot \exp \left(-0.0009 q_{c}\right)$ & 0.504 \\
\hline Quadratic & $C_{e}=2190.8-1.3604 q_{c}+0.0002\left(q_{c}\right)^{2}$ & 0.428 \\
\hline Power & $C_{e}=6194.9 q_{c}{ }^{(-246)}$ & 0.291 \\
\hline
\end{tabular}

\section{Experimental comparisons}

The results depicted in Figure 2 clearly show that the TUSC model highly underestimates the entry capacity for given circulating flow and corresponding geometric characteristics when compared with those estimated from GIRABASE. The comparison to RODEL and aaSIDRA models is more jumping and shows a high dependency by single values pairing of the entry geometry and its own circulating flow. On the contrary, the estimated entry capacities by TUSC appears generally overestimated when the German HBS method is

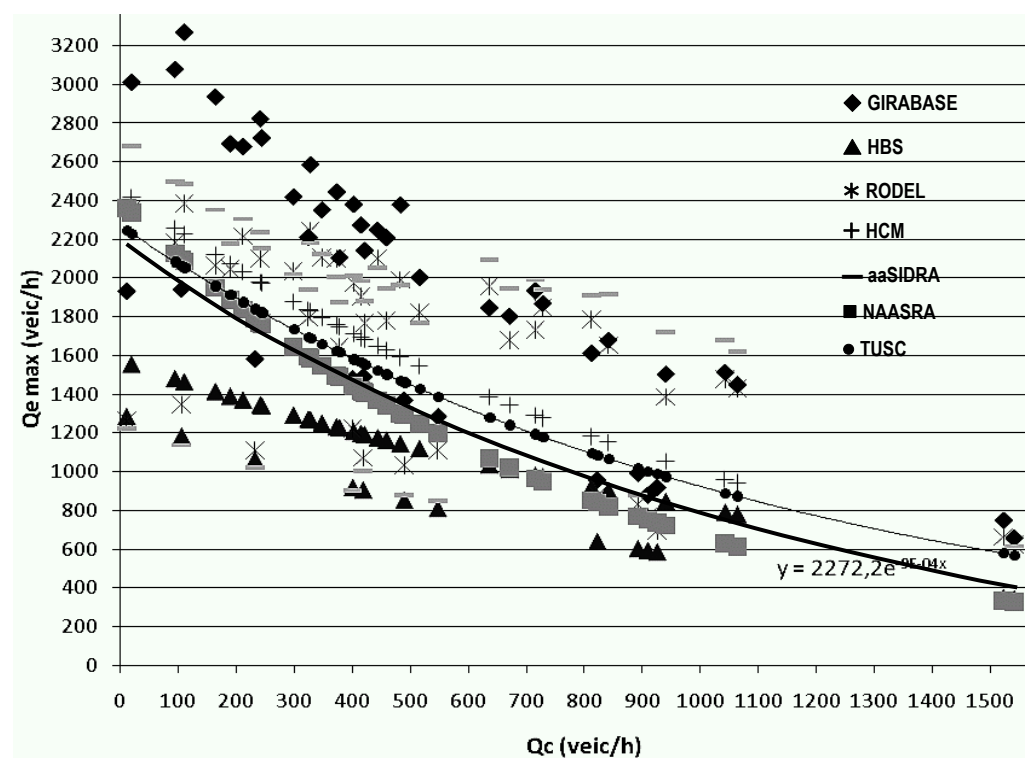

Figure 2: Developed capacity model for Italian large roundabouts, or TUSC model, along with the points generated by other six international models to the twelve roundabouts selected in Tuscany (It). 
considered. It is also interesting to draw attention to the fact that in correspondence of very high values of circulating flows either all the considered international models and, of course, the derived one lead to a lower spreading of the results. Therefore, during traffic peaks the more complicated models, such as the UK RODEL the Australian aaSIDRA that are requiring as many geometric parameters as a number of boring equations, did not show better capacity estimates than much simpler ones.

This fact has been observed previously [1] and it might be related to the drivers' behavior in approaching and crossing roundabouts at relatively low speeds during any congested period. These traffic conditions produce a very limited influence upon geometry, such as entry angle, flare length and entry radius, simply because the motorists have limited space for maneuvering and a corresponding lowered road space perception. Geometry is more influencing to the drivers during offpeaks, when they are actually forced to slow down the approaching and crossing speeds through well designed roundabout geometric features.

\subsection{TUSC versus BAHR}

The exponential equation set up in Bahrain, or BAHR model, has been applied to the twelve roundabouts sample data. The obtained entry capacity values are depicted in Figure 3 jointly with the ones derived through the previous models. Figure 3 shows also the two curves generated by TUSC equation (7) and BAHR equation (8), respectively.

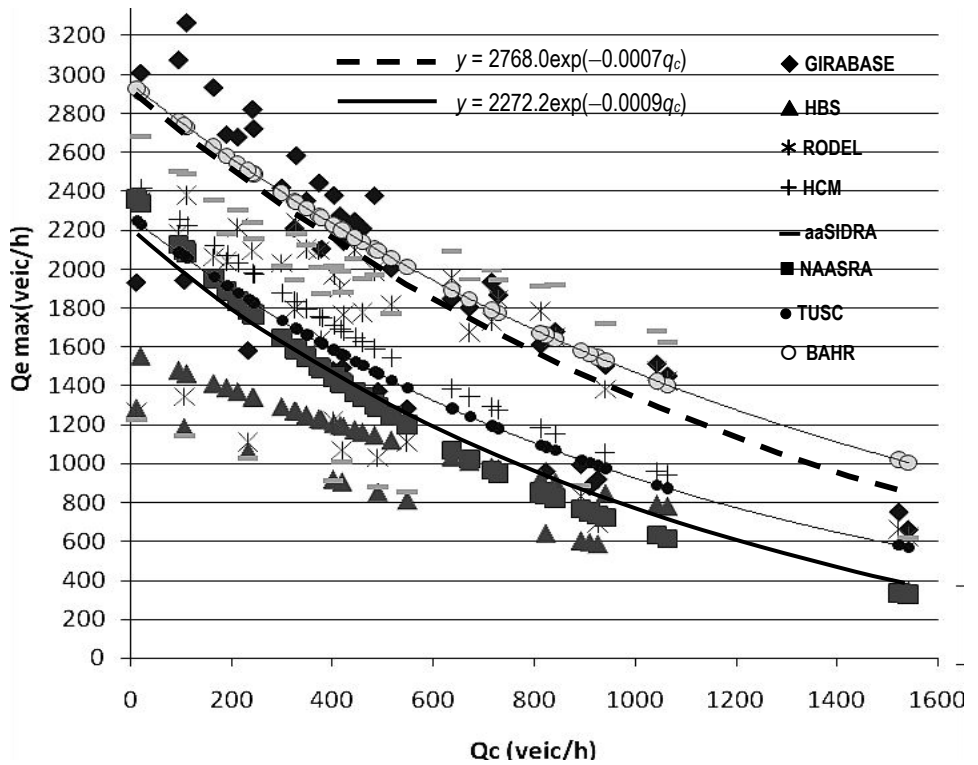

Figure 3: BAHR capacity model and TUSC model along with the sample values generated by GIRABASE, HBS, RODEL, HCM aaSIDRA and NAASRA models to the twelve roundabouts selected in Tuscany (It). 
On one side, the TUSC equation leads to values quite resembling to capacities obtained by the Australian NAASRA model, especially for instances with twolanes entry and circulating flows less than $500 \mathrm{veh} / \mathrm{h}$. On the opposite side, the BAHR model shows its major closeness to the sample estimates given by French GIRABASE when circulating flows are in the range from $300 \mathrm{veh} / \mathrm{h}$ to 1100 $\mathrm{veh} / \mathrm{h}$.

Nevertheless, one doesn't forget that the two models are obtained in respect of two fairly different sample compositions, as it is displayed in Table 3. Wide variations in capacity estimates between the two highlighted models when applied to the same measurements gathered in Tuscany, have suggested testing for their statistical significance of differences.

Table 3: Ranges of the geometric parameters measured in the sampled roundabouts in Bahrain and in Tuscany.

\begin{tabular}{|c|c|c|}
\hline Parameter & Bahrain & Tuscany \\
\hline Number of circulating lanes & 2 or 3 & 2 \\
\hline Number of entry lanes & 2 or 3 & 1 or 2 \\
\hline Inscribed diameter (m) & $63 \div 150$ & $42 \div 59$ \\
\hline Entry angle & $8 \div 32.5$ & $4 \div 45$ \\
\hline Entry radius from edge (m) & $11.5 \div 120$ & $13 \div 70$ \\
\hline Flare effective length (m) & $10 \div 55$ & $9 \div 100$ \\
\hline Entry width all lanes (m) & $6.5 \div 16$ & $4 \div 8$ \\
\hline Radius of central island (m) & $24 \div 64.5$ & $11.8 \div 21.5$ \\
\hline Width of splitter island (m) & $18 \div 82$ & $5 \div 27.8$ \\
\hline
\end{tabular}

The differences between the estimates of the two pairs constitute the sample data upon which the hypothesis of homogeneity is to be based. The homogeneity of variances is tested by computing the so called " $F$ " ratio, as follows:

$$
F=\frac{s_{T}^{2}}{s_{B}^{2}}
$$

where $s_{T}^{2}$ is the larger variance and $s_{B}^{2}$ is the smaller one.

The computed value of $F$ is then compared with the critical value, $F_{\text {crit }}$, obtained from a specific table having the desired $\alpha$ level of significance (usually $\alpha=0.05$ ) and entered in correspondence with each variance degrees of freedom, or $d . f$. [15]. If the computed (test) $F$ value is less than $F_{c r i t}$, then variances are accepted as homogeneous, and the hypothesis that the two populations are equals is adopted.

Therefore, the $F$ ratio has been computed in respect to the two capacity estimates sample pairs, one generated by the TUSC equation (7) and the other one by the BAHR equation (8) when applied to the data set related to the twelve roundabouts observed in Tuscany. The foregoing analysis of such comparison is summarized in Table 4. 
Table 4: Results of the $F$ ratio test made upon the two sample pairs generated by TUSC and BAHR equations for twelve roundabouts in Tuscany.

\begin{tabular}{|c|c|}
\hline Item & value \\
\hline TUSC sample mean & 1478.11 \\
\hline BAHR sample mean & 2098.84 \\
\hline TUSC sample variance & 177613.65 \\
\hline BAHR sample variance & 227872.45 \\
\hline Variance $d . f$. & 43 \\
\hline$\alpha$ level of significance & 0.05 \\
\hline$F$ ratio & 1.28 \\
\hline$F_{\text {crit }}$ & 1.66 \\
\hline Null hypothesis $H_{0}: s_{T}^{2}=s_{R}^{2}$ & accepted \\
\hline
\end{tabular}

Table 4 brings into prominence the ultimate fact: since the sample $F$ ratio is larger than the 0.05 level of significance tabular value $F_{c r i t}=F_{0.05,43,43}$, given in the row of $43 d . f$. and the column of $43 d . f$., the null hypothesis $H_{0}$ is accepted. So, it can be assumed that the variances came from equal populations; that is, the variances are homogeneous.

Now, the differences of population means can be tested by the Behrens-Fisher method which formulates the following hypothesis:

$$
\left|\bar{X}_{T}-\bar{X}_{B}\right|<t_{\alpha, v} \sqrt{\frac{s_{T}^{2}}{n_{T}}+\frac{s_{B}^{2}}{n_{B}}}
$$

where: $t_{\alpha, v}=$ (tabular) value of $t$-Student for the significance level $\alpha$ and degree of freedom $v=\left(n_{T}+n_{B}-2\right)$.

From the data of Table (4) the inequality (10) we obtained $\mid 1478.11$ $2098.45 \mid=619.73$ and in respect to $v=82$ and $t_{0.05,82}=1.99$; restating the hypothesis: $619.73<1.99 \times 96=191.04$ which is false.

Therefore, it must be concluded the hypothesis that the means represented by the two samples to be equal is false with $95 \%$ level of confidence, i.e. $(1-\alpha)$. When either the $F$ test or the test of means fails due to slow lack of equality, the hypothesis that populations are equal is rejected. Thus, the samples may be considered to have come from different populations. In other words, the entry capacities given by the TUSC model are not equal to the entry capacities estimated the BAHR model in respect to the same roundabout.

\subsection{TUSC Model vs. field data from Bahrain}

The TUSC model for entry capacity estimation was fit to the field data for several roundabouts with 2 entry and circulating lanes in Bahrain. As can be seen from Table 5, clear difference, of around $26 \%$, exist between the TUSC and the field data. However, the standard error of the mean of the actual field data is 
slightly higher than that for the modelled data. The paired t-test result also showed significant difference between the two (Table 6). The higher capacity results for Bahrain, when compared with TUSC, might be due to the clear differences in the geometric characteristics of the considered roundabouts and to the drivers' average headway requirements for entries. The latter is not measured here and requires further analysis. However, one may note that the average inscribed diameter for the roundabouts utilized in TUSC is around 40 to $53 \mathrm{~m}$ (see Table 1) while that in Bahrain is in the range from 63 to $150 \mathrm{~m}$. As been mentioned earlier, higher diameters might lead to better capacities up to certain limits. Comparison of headways is not considered here since it is not within the scope of the work studied here. However, it is interesting to have an experimental comparison between TUSC (i.e. Italy) and Bahrain.

Table 5: $\quad$ Comparison between TUSC modelled data and Bahrain field data.

\begin{tabular}{|c|c|c|c|c|}
\hline Parameter & mean & St. dev. & St. error of mean & sample \\
\hline Bahrain Field Data & 1014.9 & 513.7 & 83.3 & 38 \\
\hline TUSC modelled data & 748.2 & 402.6 & 65.3 & 38 \\
\hline
\end{tabular}

Table 6: $\quad$ Paired t-test of TUSC modelled results vs. Bahrain field data.

\begin{tabular}{|c|c|c|c|c|c|c|}
\hline Parameter & $\begin{array}{c}\text { mean } \\
\text { difference }\end{array}$ & $\begin{array}{c}\text { St. } \\
\text { dev. }\end{array}$ & $\begin{array}{c}\text { St. error } \\
\text { of mean }\end{array}$ & d.f. & t-test & $\begin{array}{c}\text { significance } \\
\text { (2-tailed) }\end{array}$ \\
\hline $\begin{array}{c}\text { Bahrain field - } \\
\text { TUSC modeled }\end{array}$ & 266.7 & 599.4 & 97.2 & 37 & 2.74 & 0.009 \\
\hline
\end{tabular}

\section{Conclusions}

An empirical attempt of deriving a rondabout capacity model has been made following the methodology firstly suggested and applied in Bahrein but now based on data gathered in Tuscany. This way, the original exponential model TUSC has been developed. The hypotesis of equality between the two models at hand has been tested by considering differences between the two pairs of sampled estimates given either by TUSC and BAHR models. The test fails due to the lackeness of equality of means and the two models results strongly linked to their respective data base original requirements.

Nevertheless, the present research effort should be put forward enlarging the sample sets by gathering more field observations, both in Bahrain and in Italian roundabouts.

\section{References}

[1] Al-Madani H.M.N. and Saad M., Analysis of roundabout capacity under high demand flows. Urban Transport XV, WIT Press 2009, Ashurst Lodge, pp. 223-234.

[2] Guichet B., Evolution of roundabouts in France. Proceedings of National Roundabout Conference, TRB, Colorado 2005. 
[3] Pratelli A., Design of Modern roundabouts in urban traffic systems. Urban Transport XII, WIT Press, Ashurst Lodge 2006, pp. 83-93.

[4] Fisk C.S., Traffic performance analysis at roundabouts. Transportation Research 25B, 89-102, 1991.

[5] Kimber R.M., Gap acceptance and empiricism in capacity prediction. Transportation Science 23 (2), 1989, pp. 100-111.

[6] Robinson B.W. et al., Roundabouts. An informational guide. Report FHWA-RD-00-067, U.S. Department of Transportation, Washington, 2000.

[7] NCHRP, Roundabouts in the United States, Web Only Document 94: Appendixes to NCHRP Report no. 572, TRB, 2006.

[8] Troutbeck R.J. and Kako S., Limited priority merge at unsignalized intersections. in Kyte M. (ed.) Proc. Third International Symposium on Intersections Without Traffic Signals, Portland, TRB, Washington 1997.

[9] Louah G., Panorama critique des modèles Français de capacité des carrefours giratoires. Proceedings of Roundabouts '92, Nantes, SETRA, Bagneux 1993.

[10] Kimber R.M., The traffic capacity of roundabouts. TRRL Report LR942, Crowthorne 1980.

[11] Forschungsgesellschaft fur Straßen und Verkehrswesen, Handbuch fur die Bemessung von Straßenverkehrsanlagen, HBS. FGSV Report n. 299, Verlag GmbH, Koln 2001.

[12] Akçelik R., Chung E. and Baseley M., Roundabouts: capacity and performance analysis. Rep. APR 321, ARRB, Transport Research Ltd., 1998.

[13] Transportation Research Board, Highway Capacity Manual. National Research Council, TRB special report 209, Washington 2000.

[14] Macri' F., Experimental study for a large roundabouts' entry capacity model. Laurea Thesis, Dept. of Civil Engineering, University of Pisa 2010.

[15] Snedecor G.W., Statistical Methods. The Iowa State University Press, Ames 1956. 